\title{
Local dark matter searches with LISA
}

\author{
Massimo Cerdonio ${ }^{1}$, Roberto De Pietri ${ }^{2}$, Philippe Jetzer ${ }^{3}$ and Mauro \\ Sereno $^{3}$ \\ ${ }^{1}$ Dipartimento di Fisica, Università di Padova, Italy and INFN sezione di Padova, Italy \\ 2 Dipartimento di Fisica, Università di Parma, Italy and INFN, gruppo di Parma, Italy \\ ${ }^{3}$ Institute of Theoretical Physics, University of Zürich, Switzerland
}

\begin{abstract}
The drag-free satellites of LISA will maintain the test masses in geodesic motion over many years with residual accelerations at unprecedented small levels and time delay interferometry (TDI) will keep track of their differential positions at level of picometers. This may allow investigations of fine details of the gravitational field in the Solar System previously inaccessible.

In this spirit, we present the concept of a method to measure directly the gravitational effect of the density of diffuse Local Dark Matter (LDM) with a constellation of a few dragfree satellites, by exploiting how peculiarly it would affect their relative motion.

Using as test bed an idealized LISA with rigid arms, we find that the separation in time between the test masses is uniquely perturbed by the LDM, so that they acquire a differential breathing mode. Such a LDM signal is related to the LDM density within the orbits and has characteristic spectral components, with amplitudes increasing in time, at various frequencies of the dynamics of the constellation. This is the relevant result, in that the LDM signal is brought to non-zero frequencies.
\end{abstract}

PACS numbers: $04.80 . \mathrm{Cc}, 95.35 . r d, 95.55 .-\mathrm{n}$

\section{Introduction}

According to the current cosmology paradigm dark energy and dark matter are necessary to understand the currently observed cosmological expansion and as well dark matter appears necessary to understand galactic scale phenomenology [1]. In particular the flat rotation curves of galaxies indicate that, at galactic scales, the dark matter can be some five orders of magnitude greater than the average cosmic value.

High precision Solar system tests have been providing model independent constraints on the local dark matter. The Solar system is the larger one with very well known mass distribution and can offer tight confirmations of Newtonian gravity and general relativity. What is usually investigated is the gravitational action of dark matter. Experimental bounds on non luminous matter in Solar orbit were derived either by considering the third Kepler's law [2, 3] or by studying its effect upon perihelion precession [4]. The influence of a tidal field due to Galactic dark matter on the motion of the planets and satellites in the Solar system was further investigated by [5] and [6]. The orbital motion of Solar system planets has been determined with higher and higher accuracy [7] and recent planetary astrometric data allowed to put interesting limits. The most recent analyses give an upper limit on the local dark matter density of $\rho_{\mathrm{DM}}<3 \times 10^{-16} \mathrm{~kg} / \mathrm{m}^{3}$ at the $2-\sigma$ confidence level [8, 9, 10, 11]. Such a limit falls short to estimates from Galactic dynamics [12] by 5-6 orders of magnitude. Future radio ranging observations of outer planets with an accuracy of few tenths of a meter could either give positive evidence of dark matter or disprove modifications of gravity. 
We present here, in connection with the dynamics of the drag-free LISA constellation, a new method, which has the advantage to bring the secular effects to non-zero frequencies and thus to considerably ease possible detection.

If applied to a direct gravitational measurement of the total LDM density in the Solar System, the method would be quite relevant, as it would allow to make a comparison with the presence/absence of the proposed components of local dark matter, the search for which is actively under way in a variety of direct laboratory experiments and indirect space based astrophysical observations [13]. In the present paper such a comparison at present will be made taking into consideration the diffuse GDM density, taken as uniformly distributed, but there is the possibility that the local value of the LDM at the Solar System may be somewhat [14] or even considerably higher [15], although recent numerical simulations indicate that the value of the LDM density in the Solar System should be quite close to that of the GDM diffuse density [16].

Before going to the details we notice that our method does not depend on the nature of the dark matter, whether it is baryonic or non-baryonic and behaves as cold, warm or hot dark matter as long as the dark matter has the usual gravitational interaction with ordinary matter. We assume that the dark matter is a homogeneous diffuse background of elementary particles (as the candidates which are searched for in the underground experiments such as WIMPs (the leading candidate for which is a neutralino) and axions) or at most small clumps with mass much smaller than the total dark matter mass contained in a sphere with radius of the order of the LISA orbit of about 1 Astronomical Unit, where we assume an average dark matter density equal to the LDM density. Clearly, we are mainly thinking in our proposal of dark matter in form of elementary particles as WIMPs.So our study is somewhat complementary to the analysis given in [17] for the impulsive disturbances LISA would suffer from the close encounter with dust grains and small bodies belonging to the upper mass range of interplanetary dust, with masses of $10^{-3} \mathrm{~kg}$ to $10^{15} \mathrm{~kg}$, which would apply quite the same to hypothetical dark matter clumps of that masses. Similarly if the dark matter is in the form of small-mass primordial black holes in the range of $10^{11} \mathrm{~kg}$ to $10^{17} \mathrm{~kg}$ then it has been shown that a nearby encounter of such an object with one of the LISA spacecrafts would lead to a detectable pulse-like signal [18, 19]. A difficulty is then to distinguish this class of events with those involving perturbations due to the close encounter of near-Earth asteroids and large dust grains.

\section{The method}

We use as test bed a simplified version of the planned LISA mission, which well serves the purpose of studying the basic concept. To lowest order in the eccentricities, it has been shown that the LISA $5 \times 10^{6} \mathrm{~km}$ triangular flight formation, with given choices for the heliocentric orbits, is stable and rotates rigidly around the centre of symmetry, which in turn executes a yearly revolution around the Sun, trailing behind the Earth on the same orbit [20]. We take this approximated motion of the LISA constellation as the one to be perturbed by a local diffuse matter density. In this approximation both the corrections due to higher terms in the eccentricity and all perturbations due to the Earth, the Moon, and other planets are disregarded, and as well as all the corresponding coupling effects, while in reality the constellation is expected to breathe yearly by thousands of $\mathrm{km}$ because of them. Actually such a large breathing is already present for the exact Keplerian orbits of ref. [20] in the field of the Sun alone.

To calculate the motion of a test mass in presence of an homogeneous background in 
addition to a central mass $M$ we can use [21] the Newtonian expression

$$
\phi=-\frac{G M}{r}-\frac{k}{2} r^{2},
$$

where $G$ is the gravitational constant and $r$ the distance from the central mass. The second term in the potential of Eq. (1) gives to a test mass in orbit around the Sun a perturbing central acceleration of the kind $\sim k \mathbf{r}$, with $\mathbf{r}$ the vector distance from the Sun, $r=1 \mathrm{AU}=1.5 \times 10^{11}$ $\mathrm{m}$ for LISA. We remind that the for an homogeneous background the choice of the centre of the coordinate system does not affect the result and thus we can take the Sun as the origin of it [22].

Within the current cosmological paradigm the constant $k$ would be the sum of three terms of different amplitude and sign, respectively one proportional to the density $\rho_{L D M}$ of LDM, the second one proportional to the density of cosmological dark matter and the third one related to the dark energy. As remarked above, according to current views the LDM term is at least five orders of magnitude higher than the other two terms, which therefore we disregard. In any case this shows that in principle the signals we shall discuss here would carry also information about cosmological dark matter/energy, but as it stands now the cosmological contributions appear to be masked by many orders of magnitude by the local dark matter effects. Therefore for the constant $k$ we take $k=-4 \pi G \rho_{\mathrm{LDM}} / 3$.

The $k \mathbf{r}$ perturbation can be addressed with standard Lagrange's planetary equations for the evolution of orbital elements [23]. In this framework the orbits keep on average the Keplerian behavior, but in detail there is a yearly modulation of orbital elements and it comes about a secular precession of perihelion. Lagrange's planetary equations account for the time evolution of the orbital elements of the Keplerian orbit. Let us denote the semi-major axis length, the eccentricity and the periastron argument with $a, e$ and $\omega$, respectively. Since the perturbation $k \mathbf{r}$ is radial, the inclination and the longitude of ascending node do not change, whereas the other orbital elements evolve as

$$
\begin{aligned}
& \frac{d a}{d t}=\frac{2}{n} \frac{e}{\sqrt{1-e^{2}}} k r \sin \varphi, \\
& \frac{d e}{d t}=\frac{1}{n a} \sqrt{1-e^{2}} k r \sin \varphi, \\
& \frac{d \omega}{d t}=-\frac{1}{n a} \frac{\sqrt{1-e^{2}}}{e} k r \cos \varphi,
\end{aligned}
$$

where $\varphi$ is the mean anomaly and $n \equiv \sqrt{G M / a^{3}}$ is the unperturbed Keplerian mean motion. Solving the Lagrange's equations, we get the evolution up to terms linear in the parameter $k$. The above equations can be solved keeping the orbital elements on the right-hand side unperturbed. Since the orbits of LISA satellites are going to be nearly circular we can also expand in the eccentricity.

As a worked example we study the effect, as LDM, of the accepted average GDM density of $\rho=0.3 \mathrm{GeV} / \mathrm{cm}^{3}$ [12], assumed to be diffuse and uniformly distributed as such within the Solar System. This gives $k=-1.5 \times 10^{-31} \mathrm{~s}^{-2}$ on the idealized rigid LISA orbits of ref. [20]. The $k \mathbf{r}$ perturbation operates on each of the test masses of LISA, which execute Keplerian orbits to realize the rotating triangle constellation. What is relevant here is the temporal behavior of the arm lengths $\left(L_{i j}\right)$ and of the differences between arm lengths, $\Delta L_{i j}-\Delta L_{i s}$.

The lowest approximation in eccentricity gives arm lengths $L_{0}$ that are time independent. If we now insert the perturbation, when we look for the relative motions between the test masses, we find that, after release in geodetic motion, the arms acquire under the perturbation a breathing mode, with characteristic spectral components both at the fundamental $1 y^{-1}$ frequency of revolution around the Sun, and higher frequency contributions including the 


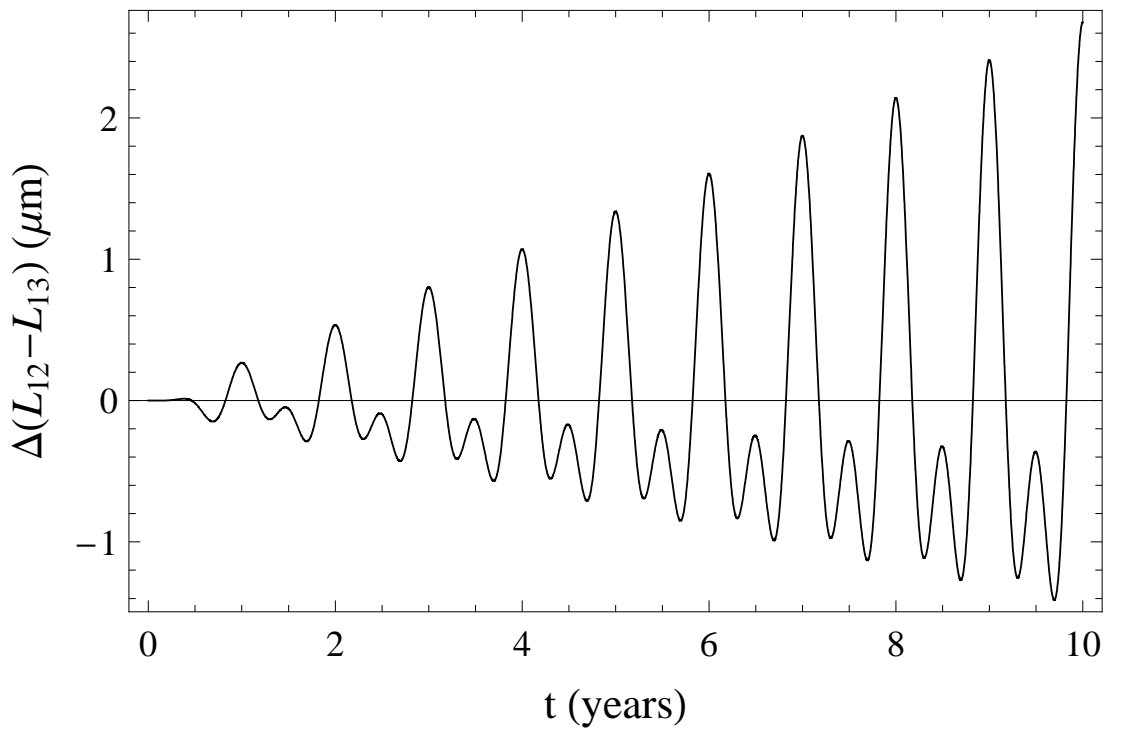

Figure 1. Differential motion due local dark matter between one pair of LISA arms after release in geodesic motion, as from the time dependence of arm lengths ( $L_{12}$ is the distance between test masses in spacecrafts \# 1 and \# 2 and similarly $L_{13}$ ); the differential motions between the other pairs are similar. The value assumed in the calculations for the diffuse LDM density is $0.3 \mathrm{GeV} \mathrm{cm}^{-3}$.

$3 y^{-1}$ frequency with which the LISA constellation returns to itself in its rotation around its barycentre. Most importantly, in the prospect of detection with TDI methods, we find

$$
\begin{aligned}
\Delta\left(L_{12}-L_{13}\right)= & -\frac{3 \sqrt{3} L_{0}}{16 n^{2}} k[n t(4 \cos (n t)+3 \cos (2 n t)) \\
& \left.+5 \sin (n t)-\frac{11}{2} \sin (2 n t)-\frac{1}{3} \sin (3 n t)\right]
\end{aligned}
$$

a time dependence of the difference between the changes in length of pairs of arms (see Eq. (5) and Fig. (1). Even more interestingly, such an amplitude modulation increases with time, giving thus to the signal a characteristic time-frequency signature. We also checked the accuracy of these results by a direct numerical integration of the equation of motion finding a perfect agreement between numerical and analytical results.

A differential motion of the order of micrometers, as in Fig. 1 1 may appear comfortably large in respect to TDI capabilities, but one should take notice of the low frequencies at which it occurs. A realistic feeling of numbers comes about as follows. The performance of LISA, in regard to the lowest residual acceleration in respect to geodesic motion, could be at best $3 \times 10^{-15} \mathrm{~m} \mathrm{~s}^{-2} \mathrm{~Hz}^{-1 / 2}$ at $10^{-4} \mathrm{~Hz}$ and possibly $10^{-13} \mathrm{~m} \mathrm{~s}^{-2} \mathrm{~Hz}^{-1 / 2}$ at $10^{-6}$ $\mathrm{Hz}$ [24], which, over a $3 y$ integration time, translates into $3 \times 10^{-19} \mathrm{~m} \mathrm{~s}^{-2}$ and $10^{-17} \mathrm{~m}$ $\mathrm{s}^{-2} \mathrm{rms}$ noise level respectively. These numbers should be compared with the value reached by the differential acceleration between the spacecrafts $k r \simeq 5 \times 10^{-20} \mathrm{~m} \mathrm{~s}^{-2}$ given by the GDM in $3 y$ of its secular increase. Of course if the LDM density would be much higher than the GDM, as proposed in ref. [15], an actual detection by LISA might appear in principle possible, but still, within the oversimplified model we analyzed, it would appear at characteristic frequencies not higher than $10^{-7} \mathrm{~Hz}$, frequencies at which we cannot expect that much from LISA in any case. 
In conclusion, we see that the effect of LDM shows in the presence of distinctive signals on the breathing dynamics that sheds light on the possibility, in principle, of detecting, or putting upper limits, on the gravitational action of LDM. However, at this stage, it is not clear if such a method would be competitive with respect to planetary upper bounds [8, 9, 10]. As we discuss in the next section, an LDM signal power may appear at distinctive frequencies where the un-perturbed contributions are small or zero. In such a framework, a few problems arise which we discuss in the next section together with their possible solutions.

\section{Discussion}

First, even in absence of other perturbations, when the rigid motion around the Sun of LISA used above is taken to higher approximation in the eccentricity [20], the arms acquire breathing modes of large amplitude. We have made analytical calculations up to 4th order in eccentricity respectively both without and with the perturbation due to LDM for the differential breathing of the arms $\Delta L_{i j}-\Delta L_{i s}$. We find that:

- without the LDM perturbation $i$ ) there is signal power at the fundamental $1 \mathrm{y}^{-1}$ and overtones only up to $7 \mathrm{y}^{-1}$, except $3 \mathrm{y}^{-1}$ and $6 \mathrm{y}^{-1}$; ii) the amplitude of the modes does not increase in time

- with the LDM perturbation $i$ ) there is signal power at the fundamental $1 y^{-1}$ and at all overtones up to $11 \mathrm{y}^{-1}$, including $3 \mathrm{y}^{-1}$ and $6 \mathrm{y}^{-1}$; ii) the amplitude of all the modes does increase in time.

So it appears that the LDM signal has a specific signature in the spectrum.

Second we must take into account the perturbation of other matter present in the Solar System, that is planets, asteroids, interplanetary dust, Solar wind. As for the gravitational effect of a local density of particles, atoms and dust, both in its steady value and in its variability due to Solar activity, it should be possible to take them in account accurately.

The distribution of the dust density is supposed to be flat centred on the Sun, with cylindrical symmetry and of thickness of the order of $1 \mathrm{AU}$, extending to some $3 \mathrm{AU}$ [25]. The value of its total density at Earth orbit, but far from the Earth so to ignore its gravitational pull, is indicated as $\rho_{\text {dust }}(1 A U)=9.6 \times 10^{-20} \mathrm{~kg} / \mathrm{m}^{3}$ [26]. We have to estimate the effect of dust and compare to that of LDM, as they may come out to be similar and hard to disentangle. The LISA constellation in its orbit stays well within an approximately uniform distribution of dust at the density value quoted above and we assume to be the same for the LDM distribution, so that we may consider LISA embedded in a spherically symmetric density of dust and LDM. This will be an extremely crude estimate, giving a conservative upper bound as the dust distribution is actually not spherical. Then the ratio between the relative accelerations among LISA test masses due respectively to dust and LDM is simply the ratio between the corresponding densities at $1 \mathrm{AU}$. If the LDM value is close to the average GDM value $\rho_{\mathrm{GDM}}=0.3 \mathrm{GeV} / \mathrm{cm}^{3}=5 \times 10^{-22} \mathrm{~kg} / \mathrm{m}^{3}$, we have that the effect of interplanetary dust on breathing motions within LISA is of the order of 200 times larger than that induced by a GDM. As for disentangling the two contributions, we see that, even if known with moderate accuracy, which, as far as it is concerned, would allow to improve by a large factor the current best limit on GDM.

The average density of electrons, protons and helium atoms is somewhat below that of a GDM. Its changes during Solar flares develop on so short time scales, in respect to the inverse of the characteristic frequencies quoted above, to be ineffective in the data analysis.

The perturbations given by Solar System massive bodies are a more serious issue. A recent calculation of LISA orbits under the perturbation of the Earth [27] shows large 
breathing modes of the arms. The analysis does not go beyond a first approximation in the ratio between the tidal effect of the Earth in respect to that of the Sun. Even preliminary (analytical) calculations appear difficult and lengthy, and it appears to us that numerical methods are needed.

We believe it would be more fruitful to our purposes to perform such calculations to see the effect on the orbits of rather the whole Earth-Moon system. Even if the breathing effect due to the Moon are very small it has a definite characteristic frequency that is $f_{E M}=8.3 \times 10^{-7} \mathrm{~Hz}$. This is the double of the frequency of of revolution of the Earth-Moon system around its barycenter and it comes out as the first non zero term in the perturbation after the monopole given by the total Earth-Moon mass at the barycentre of the system. As we have seen above, the LDM effect promotes signal amplitudes in the differential breathings at distinctive higher harmonics, where there is no signal power in absence of the LDM perturbation. If this will occur in a similar way also in the case of the Earth-Moon perturbation then the leading LDM signal may be present at, say $3 f_{E M}$, giving a unique signature at frequencies close to where LISA is sensitive.

An accurate calculation of the differential breathing of LISA due to the combined effects of the Moon motion and of the LDM diffuse density would need a full study of the LISA dynamics, aided by numerical calculations, which is beyond the scope of the present paper. We are encouraged by the present initial results and such a detailed study is under way (we outline its main features in our Conclusions below).

As discussed above the dust effect may well come out to be indistinguishable in signature and about 2 orders of magnitude larger than the effect of a GDM diffuse density. Then, if our detailed study will have any success, LISA would give limits to an LDM diffuse density at the Earth orbit no better than the limits imposed by the accuracy on independently modelling the dust distribution, that is somewhat less then 2 orders of magnitude above the GDM diffuse density. This is to be compared with the current limits on an LDM coming from the motion of the planets, which, as summarized in the Introduction, are some 5 orders of magnitude larger of the GDM value. It is worth noting that future improvements from planetary motion observations, if they concern planets within 3.5 AU from the Sun, would suffer from the same limitations from the effect of dust as well.

The notion that dark matter should be present diffusely in the Galaxy rests on the currently accepted cosmological paradigm enforced in recent years by a wealth of observational data. The value of the average GDM is $10^{5}$ higher than the value implied by cosmological expansion in the cosmological paradigm, but still it is a direct consequence of that model, when one wants to account for the rotation velocities of ordinary matter far from the centre of the Galaxy, on one side, and on the other side one wants to keep Newtonian Gravitation, as extended by Einstein with General Relativity, to hold strictly valid at the Galactic distance scale. However it has been noticed [28] that there are no independent observational grounds for such an assumption beyond the Solar System distance scale. A variety of alternative theories of gravitation have been proposed, which, while keeping valid Newton-Einstein gravity within the Solar System, allow a mild violation on Galactic and cosmological scale, with the benefit to make unnecessary the presence of any dark matter (and even dark energy). A comparative discussion of the situation says that, within the present limits given by planets motion, no definitive conclusion can be reached [8], but the possibilities of a MOND behaviour [29] within actual LISA orbits should be considered. A gravitational test like the one we propose here might give clear cut results in this respect too. 


\section{Conclusions}

The simple model shows the potential interest of the basic idea and may open the way to promising possibilities.

We need to investigate to what extend the perturbations from bodies in the Solar System would stimulate components in the relative motion between the LISA spacecrafts at the characteristic frequencies of the perturbation itself, at frequency combinations with the fundamental frequency of revolution around the Sun and at higher frequency overtones which would be distinctively identifiable in the spectrum of the the differential arms breathing. To explore this, and then in case to assess the feasibility of detections/upper limits with LISA, we need to embed the LISA test masses in the most accurate model of dynamics of the Solar System available and solve numerically for LISA orbits with and without LDM. Such a procedure will also automatically clarify the issue of the perturbations given by other massive bodies discussed above. The study should be completed by a careful evaluation of the effects of the interplanetary dust and of the Solar wind. Once understood and accounted for the effects of massive bodies, interplanetary dust and Solar activity, a last problem will concern to treat the TDI distance measurements via light propagation in a fully relativistic way [27].

\section{Acknowledgments}

In the early stage MC benefited from discussions with Michele Bonaldi, Livia Conti and Stefano Vitale. We are grateful to Fabrizio De Marchi for calling attention to the dust problem and to Oliver Jennrich for discussions. MS is supported by the Swiss National Science Foundation and by the Tomalla Foundation.

\section{References}

[1] For a most recent and comprehensive presentation of the subject and of the compelling evidence in particular for cold dark matter see e.g.: S.Weinberg, "Cosmology" (Oxford University Press 2008)

[2] J.D. Anderson et al, ApJ 342 (1989) 539

[3] J.D. Anderson, et al, ApJ 448 (1995) 885

[4] O. Gron, H.H. Soleng, ApJ 456 (1996) 445

[5] V.B. Braginsky, A.V. Gurevich, K.P. Zybin, Physics Letters A 171 (1992) 275

[6] S. Klioner and M. Soffel, Physics Letters A 184 (1993) 41

[7] E.V. Pitjeva, Solar System Research 39 (2005) 176

[8] M. Sereno and P. Jetzer, Mont. Not. R. Astron. Soc. 371 (2006) 626

[9] I.B.Khriplovich and E.V.Pitjeva, Int.Journ.Mod.Phys. D15 (2006) 615

[10] J.-M. Frere et al, Phys. Rev. D77 (2008) 083005

[11] S.L. Adler, J.Phys. A41 (2008) 412002

[12] W.-M. Yao et al., J. Phys. G 33 (2006) 1

[13] see for instance: G.Bertone and D.Merritt, Mod.Phys.Lett. A20 (2005) 1021

[14] T.Damour and L.M.Krauss, Phys.Rev. D59 (1999) 063509

[15] X.Xu and E.R.Siegel, arXiv:0806.3767 1 [astro-ph] (2008)

[16] M. Vogelsberger et al, Mont. Not. R. Astron. Soc. (in press) (2008), arXiv:0812.0362

[17] K.V. Danzmann et al, (1998) "LISA-Laser Interferometer Space Antenna", Pre-Phase A Report 2nd edn MPQ 233 (Garching, Germany: Max-Planck-Institut fur Quantenoptik) ch 4.2.5 "Disturbances due to minor bodies and dust"

[18] N. Seto and A. Cooray, Phys. Rev. D70 (2004) 063512

[19] A.W. Adams and J.S. Bloom, (2004), arXiv:astro-ph/040526

[20] S.V.Dhurandhar et al, CQG 22 (2005) 481

[21] see for instance: M.Sereno and P.Jetzer, Phys. Rev. D75 (2007) 064031

[22] R. Adler, M. Bazin and M. Schiffer, "Introduction to general relativity" (New York: McGraw-Hill, 1965)

[23] see for instance: J.M. Danby, "Fundamentals of Celestial Mechanics" (Willmann Bell 1988)

[24] P.L.Bender CQG 20 (2003) S301 
[25] R.H.Giese et al, Icarus 68 (1986) 395

[26] E.Grun et al, Icarus 62 (1985) 244

[27] S.V. Dhurandhar et al. (2008), arXiv:0805.4314 1.

[28] see for a review: P.D.Mannheim Progr.Part.Nucl.Phys. 56 (2006) 340

[29] J. Bekenstein and J. Magueijo, Phys. Rev. D 73 (2006) 103513 